number. Further search functions are proposed, such as searching and displaying by name or hospital number.

Conclusion A proof of principle system for an AR patient data clipboard was developed to connect with the FHIR database and fetch patient information for use by clinicians in a hospital environment. Further work on this system could enable the application to connect with IoT medical devices to pull patient observations in real time.

\section{CONSULTING VIRTUALLY WITH EASE: FHIR-ENABLED COMMUNICATIONS CHANNELS}

${ }^{1}$ Alexandru-Vlad Niculae, 'Dean Mohamedally, ${ }^{2}$ Neil J Sebire, ${ }^{3}$ Sheena Visram. ${ }^{1}$ UCL Department of Computer Sciences; ${ }^{2}$ GOSH DRIVE; ${ }^{3}$ University College London

10.1136/archdischild-2020-gosh.43

Introduction The COVID-19 pandemic has accelerated the uptake and adoption of virtual consultations by healthcare professionals, seeking to provide care at a distance. Standards are needed to promote a common understanding of systems, tailor-made and integrated to clinical workflows. To solve this, Health Level Seven International (HL7) have created Fast Healthcare Interoperability Resources (FHIR), a standard data exchange framework that is structured, standardised and human-readable aiming towards universal interoperability. Here, we present FHIR-enabled communication channels between patients and healthcare professionals. This Proof of Principle prototype was developed as part of a Hackathon between UCL Computer Science and Great Ormond Street DRIVE centre.

Methods Building in Python and using a synthetically generated database of patient details, we created an Application Programming Interface (API) that uses patient identifiable details in FHIR to generate unique links to Skype, then connected to the client side using Django; a Python web framework. This was developed with the capability for group calls, anticipating its application for multidisciplinary meetings and group therapy sessions for patients.

Results The resulting web application was successfully demonstrated in a testing environment with up to 500 mock patients through the FHIR API. It intends to streamline the process for initiating virtual consultations, enabling healthcare professionals to filter and select patient lists, and commence a group call over a virtual platform like Skype or Teams with a click of a button.

Conclusion The lack of interoperability between virtual platforms and the existing electronic health records (EHRs) is one challenge presented by new ways of working. Similar studies have shown that time spent logging into systems can be reduced by $75 \%$ introducing a single-system login. Likewise, we believe that FHIR-based web applications can facilitate group therapy sessions and team-based meetings in a remote and safe environment, whilst retaining a centralised health record.

\section{INTERNET OF HEALTHCARE THINGS (IOHT) HANDHELD} DEVICE FOR SECURE PATIENT DATA RETRIEVAL

${ }^{1}$ Ethan Wood, 'Dean Mohamedally, ${ }^{2}$ Neil J Sebire, ${ }^{3}$ Sheena Visram. ${ }^{1}$ UCL Department of Computer Sciences; ${ }^{2}$ GOSH DRIVE; ${ }^{3}$ University College London

10.1136/archdischild-2020-gosh.44
Introduction Fast Healthcare Interoperability Resources (FHIR), is a common tool to make it easier to read, write, and transfer medical data. Internet of healthcare things, IoHT, is a system of interrelated computing devices which are commonly used to record data about a patient, such as an ingestible sensor, which is then transmitted using FHIR over the internet. Traditionally such data is viewed as a patient care record on a computer. Here a device was developed to allow this data to be viewed in real time with a small handheld device as a faster and more convenient alternative.

Method To be faster than a digital clinical system the device uses radio-frequency identification (RFID) where each patient is provided with a small tag containing their patient number, such as a bracelet. This allows the device to only need to be near a patient to identify them. FHIR-Parser is a library to simplify the development of Python applications using FHIR, it was developed in parallel with the device to allow anyone to start building on FHIR data using familiar object-oriented programming techniques. The device was built using standard off the-shelf components and the Arduino computing platform.

Results A prototype of the device was built using an Arduino Mega, RFID-RC522, LCD Keypad Shield $(2 \times 16$ character display with 5 inputs), and a $50 \mathrm{~mm}$ by $100 \mathrm{~mm} \mathrm{3D}$ printed box. It connected over serial to a python application connected to a FHIR endpoint, successfully retrieving patient information from an id encoded RFID tag.

Conclusion Devices, such as the one demonstrated, can be miniaturised and used to display timely key care record information paperless, without the delays of a clinical information system, from an array of IoHT devices. Showing information at opportune times ensures information remains private and relevant allowing patients to receive better and more personalised care.

\section{PREDICTING LONG LENGTH OF STAY IN A PAEDIATRIC INTENSIVE CARE UNIT USING MACHINE LEARNING}

${ }^{1}$ Abigail East, ${ }^{2}$ Samiran Ray, ${ }^{3}$ Rebecca Pope, ${ }^{4}$ Mario Cortina-Borja, ${ }^{3}$ Neil J Sebire. ${ }^{1}$ UCL Centre for Doctoral Training in Al-Enabled Healthcare Systems CDT; ${ }^{2} P I C U$, Great Ormond Street Hospital; ${ }^{3}$ GOSH DRIVE; ${ }^{4}$ UCL Great Ormond Street Institute of Child Health

\subsection{6/archdischild-2020-gosh.45}

Introduction Length of stay (LOS) prediction modelling in intensive care units is a valuable capacity planning tool as hospitals attempt to clear the backlog of surgical patients resulting from the COVID-19 pandemic. Recent work in adults has demonstrated the benefits of using machine learning over statistical methods for LOS prediction, however machine learning approaches have not been applied to paediatric populations.

Objectives The study set out to develop machine learning models to predict long LOS in the paediatric intensive care unit at Great Ormond Street Hospital using electronic patient records.

Methods Paediatric intensive care patients between 1st May 2019 and 30th April 2020 were extracted from electronic patient records. Random forest, XGBoost, and multilayer perceptron models were built to predict LOS greater than three or seven days. The dataset contained demographics, ventilation data, and summary statistics of physiological time-series data, taken from the first twelve hours of admission. The 